\title{
Sui primi manuali didattici per l'insegnamento del tedesco come lingua straniera nella Venezia del XV secolo
}

La tradizione di Giorgio da Norimberga

\section{Barbara Bruzzone}

\section{CpenEdition}

\section{Journals}

Edizione digitale

URL: https://journals.openedition.org/cher/8834

DOI: $10.4000 /$ cher.8834

ISSN: 2803-5992

\section{Editore}

Presses universitaires de Strasbourg

Edizione cartacea

Data di pubblicazione: 1 décembre 2010

Paginazione: $29-46$

ISBN: 978-2-35410-029-2

ISSN: 1968-035X

\section{Notizia bibliografica digitale}

Barbara Bruzzone, «Sui primi manuali didattici per l'insegnamento del tedesco come lingua straniera nella Venezia del xv secolo», reCHERches [Online], 5 | 2010, online dal 17 décembre 2021, consultato il 24 janvier 2022. URL: http://journals.openedition.org/cher/8834 ; DOI: https://doi.org/10.4000/cher. 8834

Ce(tte) œuvre est mise à disposition selon les termes de la Licence Creative Commons Attribution Pas d'Utilisation Commerciale - Partage dans les Mêmes Conditions 4.0 International. 


\title{
Sui primi manuali didattici per l'insegnamento del tedesco come lingua straniera nella Venezia del xv secolo: la tradizione di Giorgio da Norimberga
}

\author{
Barbara BruZZone \\ Universität Passau
}

El è una bella chosa asaver todescho in questa terra. Per amor del fontego. Tu dé imparar forte. Cod. it. 261 Folio 105r

\begin{abstract}
$\mathrm{T}$ ra le prime testimonianze di attività didattica volta all'insegnamento del tedesco come lingua straniera in aree di lingua romanza, quelle dei manoscritti di Giorgio da Norimberga sono senza dubbio tra le più degne di nota. Nella Venezia del Quattrocento, vicino al Campo di San Bartolomeo, Giorgio da Norimberga insegnava il tedesco a giovani veneziani che intendevano accedere alla professione di interprete ed intermediario nelle trattative d'affari con mercanti tedeschi per conto della Serenissima. Questo articolo si propone di illustrare brevemente struttura, contenuti e tradizione di questi manuali di tedesco per gli affari ante litteram soffermandosi in particolare su alcuni tratti di indiscutibile modernità dal punto di vista didattico e su quella finestra sul mondo quattrocentesco dei mercanti a Venezia che si apre leggendo i dialoghi contenuti negli scritti.
\end{abstract}

\section{La cornice storica: Venezia e il Fondaco dei Tedeschi}

La Repubblica di Venezia ha giocato un ruolo fondamentale per il tedesco come lingua straniera poiché 
rappresentava la scuola superiore dei mercanti tedeschi provenienti dal sud della Germania. Bisognava essere stati a Venezia per valere qualcosa a casa propria.

Già nel 1308 giovani tedeschi, figli di illustri mercanti, soggiornavano a Venezia per imparare la grammatica e il saper far di conto (Simonsfeld, 1887 : 38-39, traduzione $\mathrm{BB})$.

D'altra parte gli scambi commerciali con i tedeschi erano di fondamentale importanza per l'economia veneziana poiché erano addirittura al primo posto negli affari commerciali con l'estero. Per la Serenissima divenne necessario, ad un certo punto della sua storia, cercare nuove fonti di guadagno, tra l'altro anche per finanziare la propria politica coloniale. Così, intorno al 1200, venne fondato il Fondaco dei Tedeschi. Nelle vicinanze del Campo di San Bartolomeo, i mercanti tedeschi dovevano depositare le loro merci ed alloggiare lì e in nessun altro luogo, per tutta la durata della loro permanenza nella città lagunare. Dopo il loro arrivo ai mercanti d'oltralpe venivano assegnati dei «messeti », ovvero dei sensali, che avevano il compito di seguire ogni singola transazione per evitare l'elusione delle tasse. Il messeta doveva accompagnare i mercanti durante le trattative per consigliarlo ed eventualmente anche per tradurre (Lupprian 1978:3-20). Questa nuova figura professionale, quella del sensale, creò una nuova necessità, quella di formare interpreti di professione. A fronte di queste premesse non stupisce che le primissime testimonianze dell'insegnamento del tedesco per gli affari come lingua straniera provengano proprio da Venezia.

\section{I manoscritti : tradizione e struttura}

Quando si parla dei manuali di Giorgio da Norimberga si fa riferimento ad una serie di manoscritti databili tra il $1424^{1}$ e la seconda metà del $\mathrm{xv}$ secolo. Rimando in questa sede alle ricerche di Alda Rossebastiano Bart ${ }^{2}$ che per prima si è occupata di ricostruire lo stemma dei codici in questione e della loro edizione diplomatica nella sua opera Vocabolari Veneto-Tedeschi del secolo XV (1983).

Rossebastiano Bart suddivide i manoscritti in tre gruppi. Per questa trattazione è importante precisare che i gruppi A e B sono destinati a

1 Il manoscritto porta la data 12 febbraio 1423 che corrisponde al nostro 1424 secondo il cursus veneziano che cominciava il primo di marzo

2 Alda Rossebastiano ha pubblicato per un certo periodo con il nome Rossebastiano Bart 
mercanti, mentre il gruppo C è rivolto ad un pubblico di pellegrini. Questo articolo si propone di presentare esclusivamente i primi due gruppi.

I manoscritti sono raggruppati come illustrato nella tabella qui di seguito. Le sigle riportate tra parentesi nella prima colonna verranno adottate nel resto dell'articolo:

\begin{tabular}{lcc}
\multicolumn{1}{c}{ Biblioteca di conservazione, Abbreviazione } & \multicolumn{1}{c}{ Segnatura } & Data \\
\hline Vienna, Österreichische Nationalbibliothek (WN) & Gruppo A & 1423 \\
Monaco di Baviera, Bayrische Staatsbibliothek (MN) & Cod. Ital. 261 & 1424 \\
Modena, Biblioteca Estense (ME) & Ms. ital. 405 & 1433 (1437) \\
Heidelberg, Universitätsbibliothek (HU) & Ms. Pal. Germ. 657 & Non datato (XV secolo) \\
Firenze, Laurenziana & Cod. Ashb. 352 (Frammento) & Non datato \\
\hline Sevilla, Biblioteca Colombina (SC) & Gruppo B & Non datato (XV secolo) \\
Firenze, Biblioteca Nazionale Centrale (FN) & Cod. 7.3.18 & 1467 \\
Oxford, Bodleian Library (OB) & Cod. Magl. IV 66 & Non datato \\
\hline & Cod. Canon. It. 291 & (probabilmente 1452-1467*) \\
\hline Monaco di Baviera, Bayrische Staatsbibliothek (MNa) & Cod. Ital. 362 & Cod. Palat. 1789 \\
Roma, Biblioteca Apostolica Vaticana (RV) & Gruppo C & 1460 \\
Capestrano, Convento dei Padri Francescani & Frammento & Non datato (15. Jh.)
\end{tabular}

La macrostruttura dei manoscritti dei gruppi A e B non presenta nella sostanza differenze rilevanti e può essere suddivisa in tre parti in base ai contenuti:

1. Raccolta lessicale

2. Morfologia

3. Raccolta di dialoghi

Rimando al paragrafo tre di questo articolo per ulteriori particolari.

Tutti i manoscritti sono redatti in italiano e in tedesco e possono essere utilizzati sia per l'insegnamento del tedesco che per l'insegnamento dell'italiano come lingua straniera. Le lingue sono ordinate su due colonne con l'italiano a sinistra, nella prima colonna. Dalla lingua conosciuta si arriva quindi alla lingua bersaglio.

A questo punto è necessario fare un accenno ai medievali vocabolari ex quo che contenevano la lingua volgare accanto alla lingua da imparare, 
il latino, ed avevano come obiettivo l'apprendimento di competenze comunicative e grammaticali. Si trattava di sussidi di tipo pratico la cui maggior parte veniva redatta dagli insegnanti stessi che li rielaboravano a seconda delle loro esigenze didattiche. Questi vocabulare, che non erano indirizzati a letterati bensì a principianti, costituiscono il modello sulla base del quale verranno poi compilati i manuali didattici oggetto di questo articolo (Glück 2002 : 414-416).

In che cosa consiste dunque l'elemento innovativo dell'opera di Giorgio da Norimberga? Consiste nel fatto che due lingue volgari vengono poste l'una accanto all'altra senza il passaggio attraverso il latino. Dal punto di vista linguistico, infatti, i manoscritti presentano caratteristiche dei vernacoli veneziani e toscani così come, per il tedesco, bavaresi e alemanni. L'opera di Giorgio da Norimberga è rivolta ad un pubblico non colto con l'intento di trasmettergli competenze linguistiche concrete.

Rossebastiano (1983) ha dimostrato che i manoscritti sono da ricondurre ad un archetipo che non è stato scritto a Venezia. Probabilmente Giorgio da Norimberga ha composto la sua opera prendendo a modello scritti che hanno avuto origine a Verona. Il veronese è infatti la lingua che accomuna i manoscritti dei tre gruppi. Terra d'origine dei manoscritti perduti che hanno aperto la tradizione, secondo Rossebastiano, deve essere stata la zona lungo l'Adige e non la Serenissima:

Da Verona ${ }^{3}$, probabilmente attraverso gl'inevitabili contatti derivanti dallo svolgimento di attività parallele, passarono a Venezia esempi di questi manuali, forse anche diversi dai tardi continuatori che noi conosciamo, ma certo prossimi per schema e contenuto [...]. Rielaborati fino ad assumere la struttura a noi nota, conservarono tuttavia le tracce della parlata in cui originariamente furono composti [...].

Giorgio non "inventò" [...] quest'opera pur profondamente sua, ma la compose utilizzando schemi e contenuti preesistenti. Ne fece un testo chiaramente veneziano attraverso i dialoghi, di decisa originalità, specchio mirabile del suo ambiente di lavoro dove Venezia domina attraverso il Fondaco dei Tedeschi (Rossebastiano 1983: LV).

L'opera è anonima come la maggior parte dei testi non letterari del tempo. Nonostante questo, nei manoscritti più antichi, l'autore nomina se

3 Si veda per ulteriori dettagli: Bart Rossebastiano, 1976, «Tracce di vocali turbate nel veronese del secolo XV» Atti dell'istituto Veneto di Scienze, Lettere ed Arti, Tomo CXXXIV, 635-645. 
stesso in un passaggio che, per brevità, riporto qui di seguito esclusivamente nella versione italiana omettendo quella tedesca ${ }^{4}$ :

Quanto tempo es-tu andado a scuola?

El no è anchora un anno. El serà un anno a bonaman

Per mè, tu ne sa asay in questo tempo. El basterave se tu fosse stado vinti mese in Allemagna

Ove stà to maistro?

Sul campo de San Bortolamio

Ov'è il campo de San Bortolamio?

Apresso el fontego dî todeschi

Chom'à-lo nome to maistro?

Ello ha nome maistro Zorzi

Dond'è ello,se Dio t'aida?

El è de Nurmbergo

Che homo è-llo?

Ell'è un piasevel homo

E' no digo chossì. E' digo de chi tempo è-llo

El è d'un bon aidar. El à un piasevel modo d'insignar. Senza ogni

recressimento

(MN, Folio 108 v)

Da questo passaggio si evincono diverse informazioni: Il giovane frequenta la scuola da circa un anno e il suo maestro si chiama "Zorzi", Giorgio, viene da Norimberga ed ha un modo piacevole di insegnare. Da notare inoltre il complimento che viene fatto all'allievo:«Hai imparato molto nel corso di quest'anno. È come se tu fossi stato venti mesi in Germania». Giorgio da Norimberga fa pubblicità a se stesso, al suo modo di insegnare e anche all'efficacia del suo insegnamento che promette buoni risultati in breve tempo, risultati peraltro addirittura paragonabili ad un soggiorno di studio in Germania di durata quasi doppia rispetto al corso da lui offerto. A mio avviso questa azione pubblicitaria è degna del xxi secolo. Gli argomenti addotti da Giorgio da Norimberga convincerebbero senza dubbio anche un potenziale cliente del terzo millennio a frequentare la sua scuola di lingue.

Un ulteriore passaggio ci indica inoltre che è assolutamente saggio e necessario sapere il tedesco a Venezia, per via del Fondaco.

Che imparas-tu?

E' imparo thodescho

Tu fa saviamente. El è una bella chosa asaver thodesco in questa terra.

Per amor del fontego. Tu dé imparar forte.

$(\mathrm{MN}$, folio 105r)

4 L'alternarsi dei turni in questo articolo corrisponde ad ogni nuovo inizio di capoverso, poiché i codici non contengono indicazioni al riguardo. 
Il manuale contiene anche una traccia di quei discenti che dalla Germania andavano a Venezia ad imparare l'italiano. In questo passaggio Conrad da Vienna parla con il giovane veneziano Bortolomio, che da parte sua impara il tedesco ${ }^{5}$ :

Bortolomio: Es-tu vegnudo qua per imparar latin?

Conrad: Sy io

Bortolomio : Sas-tu anchora dir «te nascha el vermochan $» 6$ ?

El è usanza che s'impara sempre la chativiera più tosto cha'l ben $[\ldots]$

Bortolomio: $\mathrm{E}$ me ne apar ben, tu à ben imparado $\quad(\mathrm{MN}, 109 \mathrm{r})$

Questo breve passaggio è solo un esempio dei motti e lazzi sparsi nell'opera, ci fa sorridere e allo stesso tempo ci rivela quanta esperienza deve aver avuto l'autore del manuale nell'insegnamento delle lingue straniere: è pur sempre vero che tra le prime cose che si imparano durante un soggiorno di studio all'estero siano proprio le parolacce. E a dir di Bortolomio, questo è un buon indicatore della conoscenza del livello di competenza linguistica raggiunta da Conrad.

La tradizione dei manuali di Giorgio da Norimberga è fortunata e continuerà fino al XVIII secolo grazie anche all'avvento della stampa che renderà il manuale di Giorgio da Norimberga un vero e proprio bestseller. La prima versione stampata è attribuita ad un tedesco, Adam von Rottweil. Nel 1477 pubblicò a Venezia una sintesi dei nove manoscritti con il titolo "Introito e Porta", un'opera rivolta agli autodidatti. Due anni dopo viene realizzato a Bologna "Il Solennissimo Vochabuolista" di Domenico Lapi. A questi incunabili che non contengono la parte dialogica e presentano varie modifiche rispetto agli originali, ne seguirono molti altri in diverse combinazioni linguistiche. Sono circa 80 le opere che sono pervenute fino ai giorni nostri. La versione più ampia contiene otto lingue diverse: il latino, il greco, il francese, lo spagnolo, il polacco, il ceco, l'inglese e il fiammingo (Rossebastiano 1984, Glück 2002, Rossebastiano 2002).

\section{Brevi note sui contenuti dei manuali}

I manuali presentano una struttura tripartita, come accennato più sopra. In questo paragrafo descriverò alcuni tratti fondamentali di ciascuna parte.

5 Per chiarezza ho aggiunto ai turni di parola i nomi degli interlocutori. L'originale non contiene tali indicazioni.

6 Traducibile con: Che ti venga la tenia, formula di malaugurio 


\section{a) La raccolta lessicale}

La raccolta lessicale contiene parole raggruppate secondo un ordine sistematico abbandonando l'ordine alfabetico dei vocabolari ex quo. I temi trattati partono da Dio per arrivare agli aggettivi di nazionalità passando per i numeri cardinali ed ordinali, alle valute e alle monete nonché a vocaboli relativi al mondo del Fondaco dei Tedeschi:

la marchadantia [la mercanzia]

el messeti [il messeta]

li messeti [i messeti]

el guadagno [il guadagno]

la perdia [la perdita] (WN, Folio 26v)

Queste liste di parole non possono comunque essere considerate semplici elenchi di vocaboli da imparare a memoria poiché l'autore propone in questa sede, per esempio, anche la declinazione dei sostantivi:

El dy - der tag

Ly dy - die tag

De dy - des tags

(WN, folio 2v)

come pure gli aggettivi e i loro gradi di comparazione, fornendo anche il loro contrario, il sostantivo relativo e delle frasi di esempio:

Zouene [giovane]

Piu zouene

Molto zouene

Massa zouene

Epiu zouene

la zouentude [la gioventù]

Vecchio [vecchio]

Piu vechio

Molto vechio

Massa vechio

Epiu vechio

la vechieza [la vecchiaia]

Tue piu vechio demi emi son piu zouene de ti [Tu sei più vecchio di me e io sono più giovane di te]

No trepar chon le vechie persone per che elli sano piu chati [Non scherzare con gli anziani perché ne sanno più di te] (WN, folio 29r)

Glück (2006:46) osserva che le parole contenute nel glossario vengono poi riproposte nella terza parte dei manuali, ovvero nei dialoghi. Si noti qui la presenza di quel principio di progressione grammaticale e lessicale 
che ancora oggi è alla base dei manuali per l'insegnamento delle lingue straniere.

\section{b) La morfologia}

Per quanto riguarda la morfologia, questa parte è dedicata principalmente alla coniugazione del verbo:

e vendo

Tu vendi

Quell vende

Nui vendemo

Vui vende

Quelli vende

[...] (WN, folio 76r $)$

I modi e i tempi trattati sono quelli usati più frequentemente nella lingua parlata ovvero, nell'ordine scelto dall'autore dei manoscritti: l'indicativo presente, l'indicativo imperfetto, l'indicativo passato prossimo, il condizionale presente, il condizionale passato e l'indicativo futuro. La scelta rivela anche in questo caso un chiaro orientamento alle esigenze concrete dei discenti che intendono acquisire competenze comunicative e non l'intero scibile relativo alla grammatica della lingua bersaglio.

\section{c) I dialoghi}

Quella parte dei manuali di Giorgio da Norimberga dedicata alla comunicazione è stata raccolta da Alda Rossebastiano nella sua opera "I Dialoghi di Giorgio da Norimberga" del 1984. Non entro qui nel dettaglio delle differenze tra i vari manoscritti e rimando per questo all'opera di Rossebastiano.

Dal punto di vista dei temi trattati i dialoghi hanno tutti un denominatore comune: rappresentano il commercio di stoffe e il mondo che ruota intorno al Fondaco dei Tedeschi ${ }^{7}$.

Eccetto in alcune sequenze che hanno luogo per la strada, la totalità dei dialoghi viene inscenata in bottega o a scuola. I dialoghi sono tra due o più persone e in molti casi il numero dei partecipanti si allarga nel corso della conversazione. Gli interlocutori sono esclusivamente uomini di diversa età.

7 In uno dei manoscritti (OB/FN), tuttavia, si parla di commercio di cavalli. Il dialetto è quello toscano, così Rossebastiano $(1983: 2)$ sostiene che questo dialogo debba aver avuto luogo lontano dalla Serenissima. 
Le donne non hanno voce nei dialoghi anche se di loro si parla, ad esempio, in alcune sequenze come quella in cui ci si riferisce alle "belle mamole" dei quartieri a luci rosse (si veda a questo proposito anche Rossebastiano $1983: 17)$.

La struttura dei dialoghi incentrati sul commercio è costante : contengono una fase di apertura con saluti e convenevoli, una contrattazione e una parte conclusiva comprendente i saluti finali. I dialoghi o le parti di dialogo che invece hanno per oggetto temi personali sono costituiti principalmente da brevi scambi di battute e danno l'impressione che il loro scopo sia perlopiù didattico perché mancano della spontaneità ma anche dell'organicità dei dialoghi modello che hanno per oggetto le trattative d'affari.

Nel paragrafo che segue entrerò nei dettagli del primo dialogo del codice ital. 261 al fine di illustrarne i contenuti e la struttura.

\section{Dialogo tra mercanti al Fondaco dei Tedeschi}

I partecipanti a questo dialogo sono in tutto tre. Nella prima parte intervengono il giovane Bortolomio, figlio di un mercante veneziano, e un mercante tedesco. Nella seconda parte si aggiunge il sensale a cui è stato affidato il mercante tedesco. I manoscritti non riportano alcuna spiegazione riguardo alla situazione né, come già accennato, una suddivisione del testo in turni di parola.

Il dialogo si apre così :

Dio te salve, Bortolomio

$\mathrm{O}$ misier, vui sì 'l ben vegnudo. El è asy ch'io no ve ho vezudo. Ove sì-vuy stado tanto ch'io no ve ho vezudo?

In qua, in là, in asay luogi

Quando vignissi-vuy?

Eri sera, da mezanote

Vegnì-vui adesso dala Magna?

Meffé sy, perché lo vuos-tu saver?

Che novelle he in Allemagna?

Bone, frar charo

E bona passe adesso in Alemagna?

Sì in tal luogo e in tal luogo no. Adoncha no se va ben seguro. El è a risego

E' muoria adesso in Alamagna?

No adesso. El è ben sta gran muoria. Ma el'è abastada. Loldà sia Dio

E' charestia in Allemagna?

El è bon marchado de pan e de vin e de ogni chossa. E' to pare in chassa?

No, el no è in questa terra 
O’ è-llo?

A Cremona

Quant'è che lui è andado?

El serà lunidì quindese zorni. El dé vegnire tosto. E' crezo ch'el sia in via anchomé

Andà-lo per terra o per aqua?

Ello andè a cavallo in là, ma el vien per aqua in qua

El è mal andar in viazo adesso in questi fangi

Vui disé vero, ma ello n'è ben usado

Ve piaserave alguna chossa?

(MN, folio 93r)

Al saluto del mercante tedesco il giovane Bortolomio risponde con l'interiezione "O misier" (Oh messere) che esprime tutto il suo stupore e il piacere nel rivedere una persona che non aveva visto da tanto tempo. Dove siete stato? Quando siete arrivato? Arrivate ora dalla Germania? Che novità ci sono? Queste domande mostrano interesse nei confronti dell'interlocutore. Interesse che viene ricambiato nella sequenza in cui il mercante si informa sul padre del giovane Bortolomio. Questa sequenza di apertura pare necessaria ed appropriata vista la distanza temporale dall'ultimo incontro. Il giovane Bortolomio passa quindi alla fase della compravendita domandando "Ve piaserave alguna chossa?». Assicura all'acquirente che sarà trattato come se fosse il padre stesso a condurre la trattativa poiché il genitore, asserisce, lo lascia agire come vuole in bottega «el me laga far chomo 'e voio chola stazon» e gli propone quindi le sue stoffe: del buon "bochasin"8 e del "valessio", sia bianchi che colorati. La propria merce viene lodata come la migliore. Come di consuetudine anche in tutti gli altri dialoghi presenti nei manoscritti, a testimonianza del modo di fare affari all'epoca, si contratta sul prezzo. Non mancano espressioni di disappunto da parte del contraente tedesco: le stoffe sono troppo care e teme di non poter raggiungere un accordo. Preferirebbe fare affari con il padre del ragazzo perché sicuramente lo tratterebbe meglio. Bortolomio gli dà ragione: il padre potrebbe regalargli tutto ma questo lui no, non può farlo. Il mercante tedesco sostiene che se lui comprasse le stoffe di Bortolomio al prezzo proposto da quest'ultimo non ci guadagnerebbe niente a rivenderle in Germania e si sarebbe preso la briga e accollato le spese per andare fino lì per niente e che se Bortolomio fosse disposto a vendergliele per 4 ducati,

8 Boccaccino: con questo nome si designa una quantità svariata di stoffe, da una tela finissima di lino o cotone, simile addirittura alla seta, fino a della tela robusta o fustagno di cotone (Rossebastiano 1984:152)

9 Valescio: tela di canapa e cotone (Rossebastiano 1984:158) 
facendogli uno sconto di un quarto di ducato, allora se ne potrebbe riparlare. Il ragazzo non si lascia impressionare e esordendo con un "Misier mio" che suggerisce un tono quasi affettuoso e comprensivo, gli dice che non si può vendere il fustagno a quel prezzo lì perché a meno di quattro ducati e un quarto non si trova niente in giro. A quel punto il mercante tedesco gli chiede se pensa che non sia possibile trovare quella stoffa anche altrove, di avercela solo lui quella stoffa. E il ragazzo dice che probabilmente sì, è possibile, ma non ad un prezzo così vantaggioso e aggiunge:

Se vui trovè chossì bon e per questo presio che vuj didi, e' voio donar el mio de bando ${ }^{10}$. Vui devì ben veder la mia roba e quando vui l'averé ben vezudo e può e' son ben contento che vui andè a zerchar in altruij. E chi ve farà meio, tolé da luj (MN, folio $95 \mathrm{r}$ )

Qui stupisce ritrovare un principio usato anche nel marketing moderno: al cliente si promette di rimborsare il prezzo della merce venduta nel caso in cui trovasse altrove lo stesso prodotto ad un prezzo inferiore. Il ragazzo suggerisce inoltre al suo cliente di osservare bene la merce che gli sta proponendo prima di andare altrove e di comprare pure da altri se questi altri gli faranno un'offerta migliore. Di fronte ad un'argomentazione così ferrata e alla fiducia nella qualità del proprio prodotto il mercante tedesco capitola e osserva che il ragazzo ha parlato da "valent'homo" e che si fida di lui perché «a dir lo vero e' no me intendo ben del valessio né del bochassin». Il ragazzo gli dice che lo tratterà come se stesse comprando le stoffe per se stesso. A quel punto il mercante annuncia che intende andare a desinare e tornare in seguito con il suo messeta. Il ragazzo prima si dichiara d'accordo e si congeda ma subito dopo lo richiama e gli offre qualcosa da bere. Il tedesco obietta che è troppo presto per bere. Bortolomio commette a quel punto un passo falso meravigliandosi che il mercante non accetti il suo invito poiché i tedeschi, si sa, notoriamente bevono volentieri ${ }^{11}$. Il tedesco esprime il suo disappunto:

Dì pur anche ti chossì, che li todeschi sien imbriagi. E hi taliani no falla miga quando elli se ne abati ${ }^{12}$. Ben che li todeschi ebia la nominanza (WN, 95v)

10 De bando: gratuitamente

11 Riguardo al consumo di bevande alcoliche dei tedeschi si vedano anche Simonsfeld $1887: 17$, Pausch 1972:63

12 Se ne abati: nel caso in cui ne abbiano l'occasione, gli si presenti l'occasione 
Dopo aver proferito questa frase però chiede che cosa c'è di buono. E allora gli vengono offerti diversi tipi di vino e pane dolce sul tagliere ${ }^{13}$. La parentesi conviviale presenta anche un passaggio che potrebbe essere interpretato come un esempio del noto e ancora valido atteggiamento italiano del "far complimenti". A chi tocca prendere il bicchiere colmo di vino per primo? Secondo quali regole di precedenza?

Tolè suso

Chomenzè in lo nome de Dio

E' no farò zerto

Tolé suso per amo $<\mathrm{r}>$ de compagnia

E' no farò certo avanti de vui seguramente

Negun farà avanti de vuy, questo io ve so ben dire

Mo via, el è meio esser vilan cha esser recressevele ${ }^{14}$

(MN, 96r)

E alla domanda se il vino è di suo gradimento l'ospite tedesco risponde affermativamente aggiungendo che gli piace non solo il vino ma anche tutto ciò che possiede il giovane veneziano e che il mercante veneziano è ancora migliore del vino. A questo complimento il giovane non risponde ringraziando, come ci si aspetterebbe da un interlocutore moderno. Ma pur sempre con l'offerta di fargli avere altro vino in qualsiasi momento lui lo desideri.

Al termine della parentesi conviviale il tedesco si congeda da Bortolomio il quale risponde al saluto di commiato ricordandogli di portare con sé il suo messeta poiché in caso contrario non riusciranno a concludere niente ${ }^{15}$. Subito dopo gli propone di desinare con lui. Il mercante tedesco declina l'invito con una risposta che ai nostri occhi potrebbe sembrare troppo diretta, anche perché non disponiamo di informazioni riguardo a gesti e intonazione: «No, voglio desinare al Fondaco».

Il dialogo si interrompe qui e continua più tardi con una sequenza in cui il mercante tedesco torna da Bortolomio. Si deduce che sia passato un giorno, nel frattempo, infatti il padre del giovane veneziano, come si viene a sapere da Bortolomio, è rientrato a casa. Il messeta arriva con il mercante tedesco e il dialogo inizia con un «Bortolomio, dove sei ?» a cui segue «Sono qui» (a questo punto il lettore si immagina che il sensale e il suo mandatario

13 Nel manoscritto si parla di «confetto", probabilmente pane di confetto ovvero focaccia dolce.

14 Recressevele: fastidioso.

15 Lupprian (1978: 16) scrive che di fatto era proibito ai mercanti tedeschi trattare gli affari senza la presenza del sensale attribuitogli al suo arrivo secondo le disposizioni in merito del 1317 e del 1346. 
siano entrati nella bottega deserta e da qualche parte provenga la voce del giovane) e prosegue immediatamente con qualche sentore di conflitto senza scambio di saluti né di presentazioni:

Sì-vui vegnudo per quel fato?

Meffé sì, no lo sas-tu?

Volì-vuy che ve mostra el fustagno?

Sì, monstra za ogni chosa

Perzò semo nui vegnudo qua

No ve choruzà cho mi. E' vorave inanzi che quanti ge n'è in Venesia se choruzassenn cho mi cha vuy. E' son pur vostro amigo, se vui volé o no

$(\mathrm{MN}, 96 \mathrm{v})$

Il tono della trattativa continua invariato arrivando addirittura ad uno scambio di frasi dalla carica decisamente aggressiva in cui si scende sul personale criticando apertamente l'interlocutore. Il problema è sempre lo stesso: il prezzo è troppo alto.

E’ no vidi mai chossì strani'homo chomo vui sidi

E mi no viti may el to simel

Tu no vol che nesun favela

Le sé tranta peze, dî qual e' voio zento e duose ducati

Le sé massa charo, e' li farò haver in altrui per meno

Questo può ben esser, ma el no die esser chossì fata roba

Perdonème, el so se semeia a quello de questa terra

Chi vol have bona roba la chonvien pur pagar

El no la vole de bando

(MN, folio 97v)

Bortolomio rimane fermo sulla sua posizione e non accetta l'argomento che altrove quella stessa stoffa costa di meno.

E allora il messeta cambia tono e cerca di convincerlo dicendogli che il suo mandante lo pagherebbe con «ducati de zecha». Così Bortolamio cambia tono a sua volta e appellandosi al buon senso del suo interlocutore, gli dice che glielo accorderebbe volentieri il prezzo richiesto ma che allora non ricaverebbe nulla dalla vendita per via delle spese di trasporto da Cremona e che la sua bottega dovrà pur registrare un guadagno. A questo punto il messeta spiega che vorrebbe semplicemente un prezzo equo ed uguale a quello concordato a tutti gli altri insinuando quindi che il giovane veneziano non si stia comportando correttamente. Bortolomio gli assicura che gli sta concordando un prezzo addirittura inferiore a quello che pretenderebbe da chiunque altro e sembra voler capitolare dicendo di sentirsi messo alle strette e che non osa parlare in presenza del messeta per paura che si metta 
ad urlare e aggiunge che in ogni modo sono sempre i poveri mercanti a perderci poiché i sensali e i loro mandatari stanno dalla stessa parte:

El no sa may far marchà cho mi, se non chom bataia

Questo sì è per la tua despiaseveleza, la quale tu usa sempre cho mi. El marchadante dise pur chossì

Vui ve chognossé ben insembre. Vuy no ve morsigerì miga l'un l'altro. Ma nui poveri merchadanti son quelli che ànno el danno

$(\mathrm{MN}$, folio $97 \mathrm{r}$ )

Dopo questa lamentela segue una sequenza nella quale i contraenti si danno la mano per giungere finalmente ad un accordo: quattro ducati e un quarto al pezzo di stoffa ovvero il prezzo di partenza. A questo punto il messeta cambia strategia e in un tentativo in extremis di ottenere lo sconto passa ad un'aperta minaccia:

Tu ge chonvien lassar quell quarto

Azo ch'el vegna un'altra volta oiù volentieri da ti

A quel punto il giovane Bortolomio temporeggia e dice che deve prima chiedere al padre e dice di dover andare a chiedere a lui se è d'accordo. Il messeta prima gli dice di sbrigarsi e poi lo richiama : «Oldis-tu? Varda se lu instesso po' vegnir zosso» (MN, folio 98r). Poiché sarebbe meglio accordarsi con il padre visto che il giovane è «duro».

Il padre però non può scendere, così dice Bortolomio, perché è ancora stanco dalla cavalcata del giorno precedente e aggiunge che comunque lui è d'accordo con quello che dice il figlio e che gli rincresce che il messeta e il suo mandante siano così adirati con lui e che le loro sono pure pretese di tirare sul prezzo e ottenere merce di buona qualità sottoprezzo. A quel punto il messeta reagisce con un diretto «tu ha torto adesso» al quale Bortolomio ribatte con un «ho ragione, in buona fede».

E qui si inserisce il mercante tedesco che pone la domanda:

El sa chostù che nui marchadassemo anchuò fustagno in una stazon ch'io no te voio menzonar? Alora ne fo mostrado fustagno che me parea chossì bon chomo questo e anchora mejor pur assay. E si me diseva la peza pur per quatro duchati. Finalmente el me l'averave lagado per quatro duchati men un quarto

(MN, folio $98 \mathrm{v}$ )

ritornando quindi all'argomento del prezzo migliore che si sarebbe ottenuto da un'altra parte.

Bortolomio pare spazientirsi e gli domanda a quel punto perché non ha acquistato la merce altrove e aggiunge che se lo avesse fatto avrebbe fatto un favore anche a lui ed esprime stupore e stizza riguardo all'insistenza a 
voler comprare le stoffe proprio lì da lui. Tuttavia fa un ultimo tentativo di convincere il mercante scucendo la stoffa per dimostrarne la robustezza e la qualità.

A quel punto quest'ultimo reagisce con una minaccia: dammi il fustagno e non lasciare che io vada via che se me ne vado non torno più. Il tentativo di intimidazione però fallisce perché il ragazzo non si lascia impressionare e dice che il prezzo è quello, prendere o lasciare, e che in fondo non si preoccupa proprio di non riuscire a vendere altrimenti la sua merce poiché è di buona qualità.

La conclusione della contrattazione si intravede dopo qualche battuta: il mercante tedesco chiede finalmente al suo sensale di riferire al ragazzo che intende acquistare la merce. Lui e Bortolomio si porgono la mano e concordano sui quattro ducati e un quarto. Bortolomio si lamenta dicendo che stavolta l'hanno proprio stremato e che «Vui sidi stado do chontra mi solo» ( $\mathrm{MN}$, folio 99v). Aggiunge però che spera comunque in futuro di poter far affari con loro e con i loro amici e che suo padre si augura che loro raccomandino ad altri la sua bottega. Il messeta dice che senz'altro lo farà ma non per lui bensì per suo padre perché

Tu ne ha stentado tuto anchuò chostù e mi con questo puocho de fustagno. E' ho zà chomprado per mille ducati più tosto. Tu è scarso ${ }^{16}$ adesso, e si è zovene, chomo farà-tu quando serà vechio?

$(\mathrm{MN}$, folio $99 \mathrm{v})$

Dopo aver espresso questa lamentela e questo commento sulla tirchieria del giovane, si passa alla conclusione dell'affare pattuendo una caparra e organizzando il trasporto della merce acquistata. Vengono mandati a chiamare quattro o sei "bastasi" 17 al Fondaco. La sequenza successiva riguarda l'acquisto di cotone. Qui il messeta chiede la "zonta" ovvero il buon peso in omaggio. Bortolomio dice che normalmente per il cotone non si dà niente in omaggio e commenta su una caratteristica comune ai tedeschi:

El è usanza di thodeschi che elli chonvien sempre aver zonta In bona fe, el parave a un todescho, ch'el no avesse fatto marchà, s'el no pavese zonta (MN, folio $100 \mathrm{v})$

Questa volta apparentemente senza suscitare alcuna reazione nei suoi interlocutori che passano al calcolo delle somme dovute e alla trascrizione del debito sul banco:

16 Scarso : tirchio

17 Bastasi: facchini 
Vien via che te farò schriver novanta ducati in bancho

E diese has-tu abudo caparra, che vien zento ( $\mathrm{MN}$, folio 100v)

Alla fine delle sequenze dedicate ai calcoli, Bortolomio invita i suoi clienti a brindare all'affare concluso: "Aspetè, nuj devemo bevere del marchado" (MN, folio 100v). Dopo la parentesi conviviale Bortolamio coglie l'occasione per chiedere al mercante tedesco se ha intenzione di comprare del boccaccino. Questi risponde di non aver più soldi. Bortolomio gli offre di fargli credito fino alla volta successiva a patto che lui si impegni per iscritto a restituire il denaro. Il mercante tedesco rifiuta l'offerta dicendo che prima preferirebbe vendere la propria merce e aggiunge di non saper comprare a credito, che non è sua abitudine e che non vuole impegnarsi a farlo neanche in quella occasione.

Il dialogo si conclude qui e il lettore ritorna dal suo viaggio nel tempo dopo esser stato trascinato nella Venezia del '400: la contrattazione viene inscenata infatti in maniera talmente vivace ed avvincente che facilmente ci si può immaginare che sia una trascrizione di una dialogo che realmente ha avuto luogo. Dal punto di vista linguistico, le frasi sono brevi e ricche di elementi tipici della lingua parlata (Holtus/ Schweickard 1985: 354-356) ${ }^{18}$. L'autore del dialogo, inoltre, sembra sapere esattamente sia come si svolgevano le trattative sia quali erano le regole vigenti riguardo alla «messettaria». Attraverso i suoi dialoghi-modello il discente nonché il lettore dei nostri giorni impara non solo la lingua ma anche come comportarsi nelle trattative, ovvero lo script relativo all'acquisto in bottega tra grossisti. Lo script potrebbe essere riassunto in questo modo:

1. scambio di saluti

2. small talk

3. presentazione della merce

4. contrattazione del prezzo

5. accordo sul prezzo

6. brindisi

7. saluti di congedo

La fase dedicata alla presentazione della merce nel dialogo di Giorgio da Norimberga è, rispetto ad una contrattazione moderna molto ridotta, anzi pressoché assente. La parte dedicata alla contrattazione, invece, è piuttosto prolissa e il giovane veneziano comunque non cede sul prezzo che alla fine

$18 \mathrm{Si}$ veda a questo proposito anche il lavoro di Holger Klatte, 2008, Gesprochenes Frühneuhochdeutsch in tschechisch-deutschen Sprachbüchern, Kümmerle Verlag, Göppingen. 
rimane quello iniziale. Le argomentazioni del sensale per ottenere uno sconto paiono deboli poiché vertono quasi esclusivamente sul fatto che la merce si può reperire altrove ad un prezzo più vantaggioso.

La seconda parte del dialogo in cui è presente il messeta rivela una forte carica di conflittualità al contrario della parte in cui Bortolomio conversa da solo con il mercante tedesco. Le trattative, come già accennato, dovevano essere concluse alla presenza del messeta, così dettavano le leggi della Serenissima. La prima parte del dialogo rappresenta quindi una conversazione con scopi diversi rispetto a quelli perseguiti nella seconda parte. Alzare la voce, criticare e minacciare il proprio interlocutore sembra lecito in questo caso. Questa "lite" ha con tutta probabilità un valore rituale e fa parte dello script relativo alle contrattazioni, forse anche facilitato dal grado di confidenza tra il sensale veneziano e il giovane venditore che si conoscono perché conducono di frequente trattative ("Questo sì è per la tua despiaseveleza, la quale tu usa sempre cho mi», MN, 97r).

Dal dialogo emergono chiaramente sia la funzione di controllo del messeta così come i rituali legati alla conclusione di una trattativa d'affari che nel mondo degli affari italiani del terzo millennio non sono molto cambiati: la convivialità, il voler stabilire in qualche modo un rapporto personale con il proprio potenziale cliente invitandolo per esempio a pranzo è anche un elemento chiave del mondo delle trattative contemporaneo.

Dal punto di vista didattico si può osservare quanto moderne siano le riflessioni del nostro autore riguardo all'acquisizione delle competenze comunicative che prendono a modello dialoghi con tutta probabilità realmente condotti.

\section{Bibliografia}

Glück Helmut, 2002, Deutsch als Fremdsprache in Europa vom Mittelalter bis zur Barockzeit, Berlin, Walter De Gruyter.

Glück Helmut, 2002, Die Volkssprachen als Lerngegendstand im Mittelaler und in der frühen Neuzeit, Berlin, Walter De Gruyter: 113-124.

Glück Helmut und Bettina Morcinek, 2006, Ein Franke in Venedig, Wiesbaden, Harrassowitz.

Holtus Günter und Wolfgang Schweickard, 1985, «Elemente gesprochener Sprache in einem venezianischen Text von 1424: "Das Italienisch-Deutsche Sprachbuch von Georg von Nürnberg" " in: Holtus, Günter und Edgar Radke (Hrsg.) 
Gesprochenes Italienisch in Geschichte und Gegenwart, Tübingen, Günter Narr: 354-376.

Karnein Alfred, 1976, «Deustch als Fremdsprache im 15. Jahrhundert: Das Sprachbuch Meister Jörgs» in: Wierlacher, Alois (Hrg.) Jahrbuch Deustch als Fremdsprache, 2: 1-13

Luprian Karl-Ernst, 1978, Il Fondaco dei tedeschi e la sua funzione di controllo del commercio tedesco a Venezia, Venezia, Quaderni 6.

Pausch Oskar, 1972, Dasälteste italienisch-deustche Sprachbuch, Wien, Österreichische Akademie der Wissenschaften.

Rossebastiano Bart Alda, 1983, Vocabolari Veneto-Tedeschi del secolo XV, L'Artistica, Savigliano.

Rossebastiano Alda, 1981, «Per la storia dei vocaboli italiano-tedeschi. Localizzazione e datazione di un ramo della tradizione manoscritta», in: La Ricerca Dialettale III: 289-302.

Rossebastiano Bart Alda, 1984, Antichi vocabolari plurilingui d'uso popolare: la tradizione del 'Solenissimo Vochabuolista', Alessandria.

Rossebastiano Bart Alda, 1984, I “Dialoghi” di Giorgio da Norimberga, L’Artistica, Savigliano.

Rossebastiano Alda, 2002, «Deutsch-italienische Vokabulare des 15. Jahrhunderts » in: Glück Helmut, Die Volkssprachen als Lerngegendstand im Mittelaler und in der frühen Neuzeit, Berlin, Walter De Gruyter: 1-20.

Simonsfeld Henry, 1887, Der Fondaco dei Tedeschi in Venedig und die deustchvenetianischen Handelsbeziehungen, Cotta, Stoccarda. 